\title{
Molecularly imprinted electrochemical sensor for the point-of-care detection of a breast cancer biomarker (CA 15-3)
}

\author{
João G. Pacheco*, Marta S.V. Silva, Maria Freitas, Henri P.A. Nouws, \\ Cristina Delerue-Matos \\ REQUIMTE/LAQV, Instituto Superior de Engenharia do Porto, Politécnico do Porto, Rua Dr. António Bernardino de Almeida 431, 4200-072 Porto, Portugal
}

\author{
Keywords: \\ Breast cancer \\ Molecularly imprinted polymer \\ CA 15-3 \\ Electrochemical sensor \\ Point-of-care analysis
}

\section{A B S T R A C T}

The incidence of breast cancer has been increasing over the years. To control and monitor this disease several tumor biomarkers have been proposed for early diagnosis, patient follow-up and/or treatment guidance. The only serum breast cancer biomarker in current use is the cancer antigen 15-3 (CA 15-3). In this work a molecularly imprinted polymer (MIP)-based electrochemical (voltammetric) sensor to monitor breast cancer was developed, based on direct surface imprinting of CA 15-3 on a screen-printed gold electrode (Au-SPE). The imprinting was performed in two steps: (1) adsorption of CA 15-3 on the surface of the Au-SPE and (2) electropolymerization of 2-aminophenol around the adsorbed protein. After extraction of the imprinted protein voltammetric analysis was conducted using hexacyanoferrate(II/III) as redox probe, measuring the signals before and after protein binding. The sensor was characterized by voltammetric techniques and electrochemical impedance spectroscopy, and the analytical responses of imprinted and non-imprinted polymer sensors were studied. A linear relationship between the peak current intensity of the redox probe and the logarithm of CA 15-3 concentration was established between 5 and $50 \mathrm{U} \mathrm{mL}^{-1}$, achieving a limit of detection of $1.5 \mathrm{U} \mathrm{mL}^{-1}$. The prepared MIP-sensor provides fast ( $15 \mathrm{~min}$ ) analysis and is cheap, easy to prepare, disposable and could easily be integrated in small portable point-of care devices.

\section{Introduction}

Cancer has been a constant challenge for science and medicine and is a major public health problem. In 2012, the International Agency for Research on Cancer (IARC) estimated that, worldwide, there were 14.1 million new cancer cases, 32.6 million people were living with cancer (within 5 years of diagnosis) and 8.2 million people died from cancer [1]. The number of new cases is expected to rise by about $70 \%$ over the next two decades [2]. Cancer is, as defined by the World Health Organization (WHO), a generic term for a large group of diseases that can affect any part of the body. "One defining feature of cancer is the rapid creation of abnormal cells that grow beyond their usual boundaries, and which can then invade adjoining parts of the body and spread to other organs, the latter process is referred to as metastasizing." [2]. In metastasis, which is the major cause of cancer death, cells lose their adhesion capacities and migrate to healthy tissues.

\footnotetext{
* Corresponding author.

E-mail address: jpgpa@isep.ipp.pt (J.G. Pacheco).
}

Breast cancer, with 1.67 million new cases in 2012 , ranks as the second most common cancer and as the fifth most common cause of death from cancer [3]. The development of screening programs and improvements of treatment and follow-up have been able to lower the mortality in the past few years, however it is still the leading cause of cancer-related mortality in women [4]. Therefore, several challenges still remain, including diagnosis, definition of high risk recurrence population, early diagnosis of recurrence and guidance through the different lines of treatment in the management of metastatic disease [5]. Important research has been conducted to identify suitable biomarkers of breast cancer, which has led to advances in its detection and treatment [6]. These biomarkers can be one of several substances that are produced by cancer cells, and their levels can be monitored in tumor tissues or biological fluids such as blood, urine, serum, plasma, stool, sputum or nipple discharge. Many breast cancer biomarkers have been suggested, but only CA 15-3 has been routinely adopted as a potential predictor of treatment failure in the metastatic setting [4]. A decrease or return to normal levels of this biomarker may indicate that the cancer has responded favorably to therapy [7-9]. 


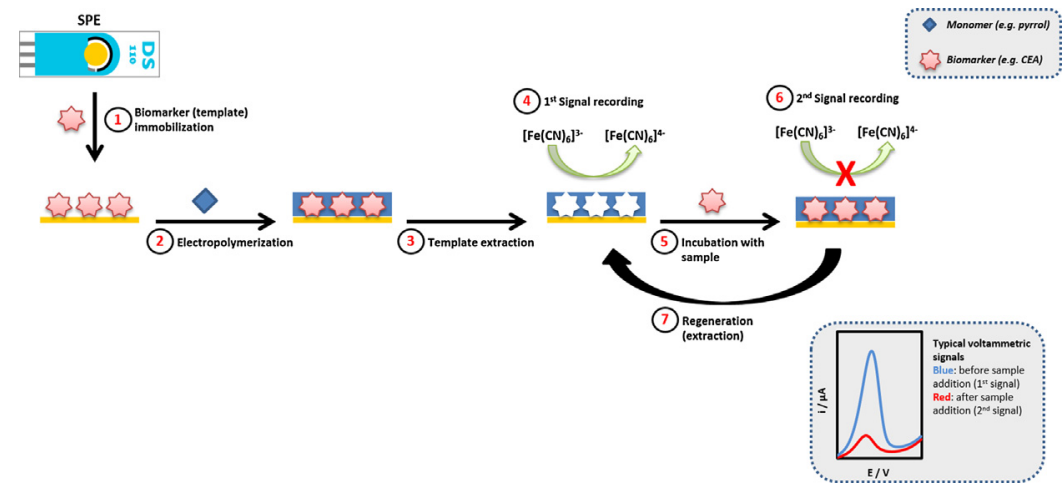

Fig. 1. Schematic illustration of the construction and operation of the MIP/Au-SPE.

A

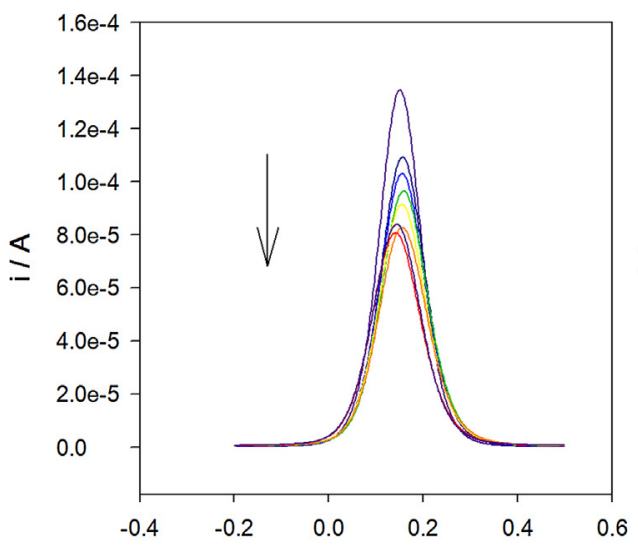

Potential / V

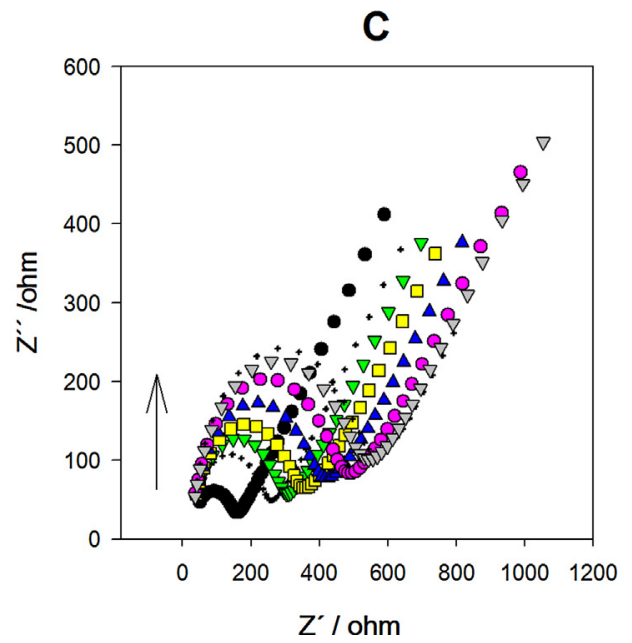

B

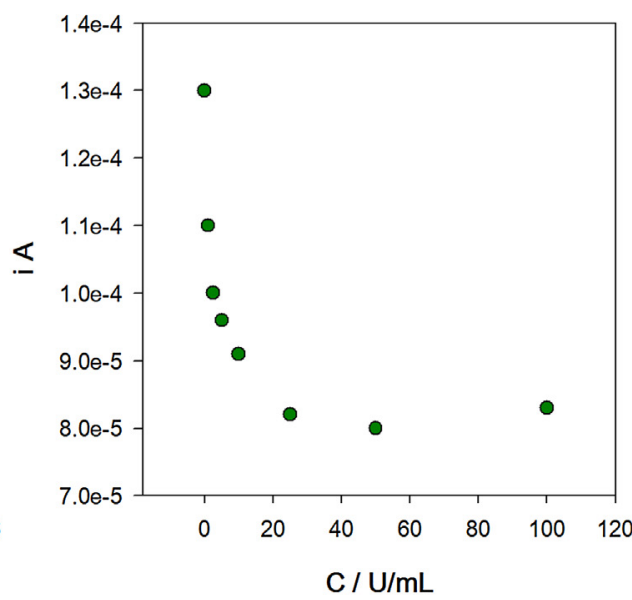

D

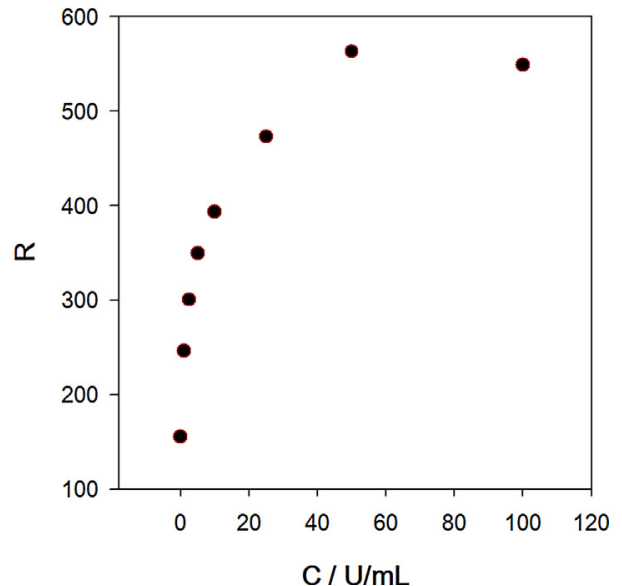

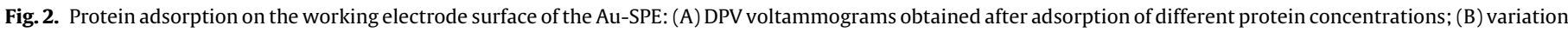

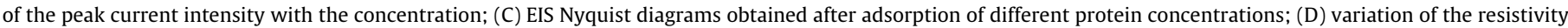
with the concentration. CA 15-3 concentration: $\left(0-100 \mathrm{U} \mathrm{mL}^{-1}\right)$.

Electrochemical molecularly imprinted polymer (MIP)-based sensors, and especially the ones using voltammetric detection, can be adequate tools because of their selectivity and sensitivity which could allow early detection of many diseases. Generally, MIPs are obtained by bulk (3-D) polymerization, a process in which selected functional monomers (either organic or inorganic materials) are polymerized around a target analyte (template) in the presence of a crosslinking agent. After polymerization, the template molecule is extracted and a polymer matrix, with sites complementary in shape, size and functionality to the imprinted molecule, is obtained $[10,11]$. Like this, MIPs show favored affinity towards the template molecule compared to other molecules [11]. The advantages of MIPs are their robustness, stability to changes in chemical and thermal conditions, ease of preparation, reusability and low cost. In principle, any molecule, from very small to macromolecules, like proteins or even cells, can be subject to imprinting in dif- 

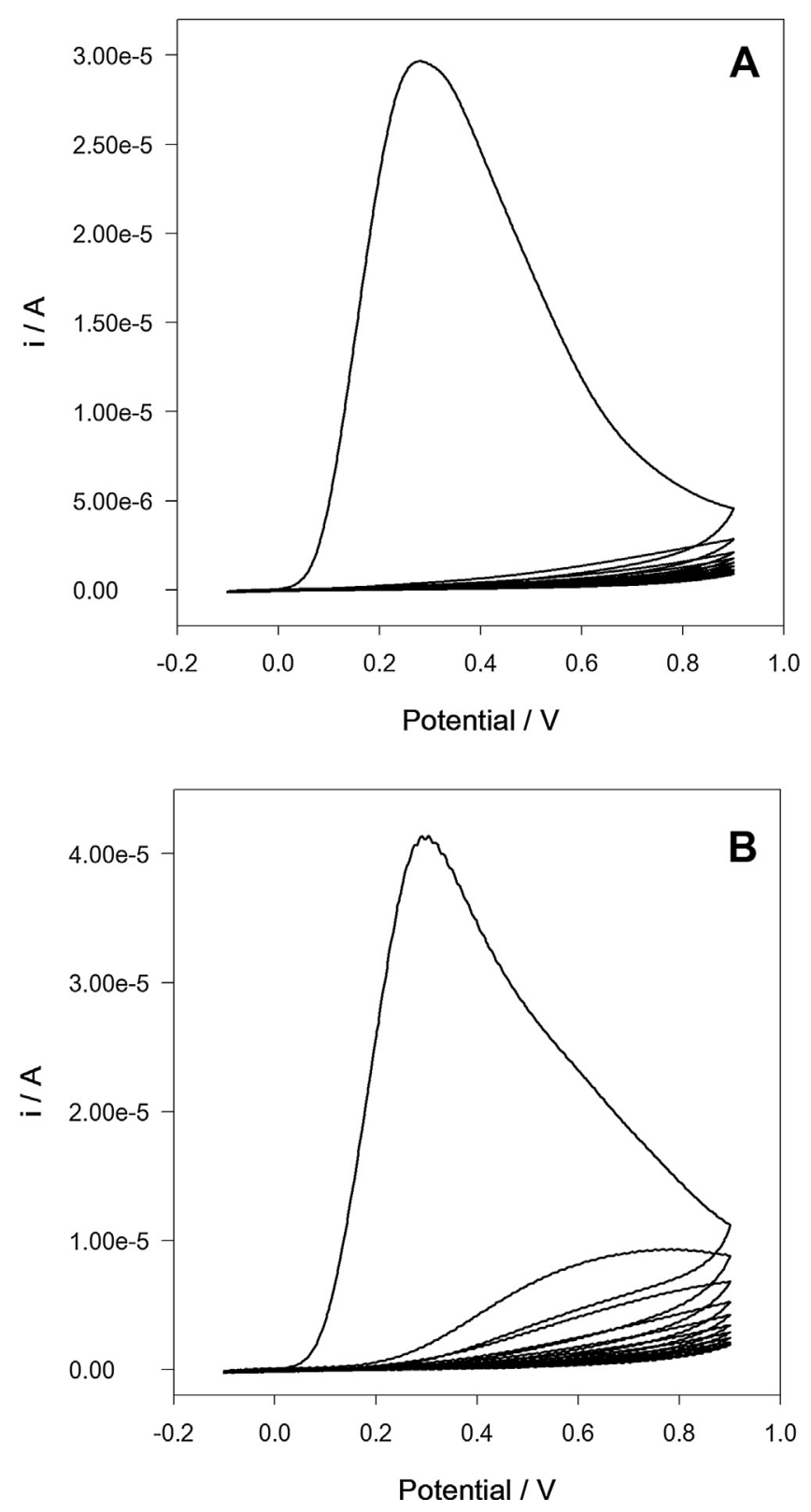

Fig. 3. $\mathrm{CV}$ voltammograms recorded during the electropolymerization of 2aminophenol (10 mM in $0.1 \mathrm{M}$ phosphate buffer $\mathrm{pH} 7.4$ ). (A) NIP/Au-SPE and (B) $\mathrm{MIP} / \mathrm{Au}-\mathrm{SPE}$.

ferent matrices [12]. Nevertheless, imprinting of macromolecules (like proteins) is problematic and challenging and its development has been slower compared to the wide range of successful applications for small molecules [13]. Several problems have been identified in the molecular imprinting of proteins. These problems are mainly related to their size (poor mass-transfer and permanent entrapment), complexity, conformational flexibility, and solubility (limited choice of solvent). Surface imprinting (2-D) has proven to be a good approach for macromolecule imprinting. Using this procedure thin film polymers, with good control of their thickness and morphology, can be produced $[14,15]$. The imprinted binding sites are located at or close to the surface of the MIPs, which solves the problems of restricted mass transfer, enabling easy extraction and rebinding [14]. Although there are several reports about molecularly imprinted polymer sensors for protein detection [16-18], few are developed for cancer-related protein detection [19].
In this work a voltammetric MIP sensor for the analysis of CA 15-3 was developed. The MIP production was based on direct surface imprinting of the biomarker protein on the surface of a screen-printed gold electrode (Au-SPE) using eletropolymerization. The reduced size of the SPEs lowers the sample volume down to $30-40 \mu \mathrm{L}$, which is advantageous especially in the analysis of biological samples. Furthermore, SPEs can easily be integrated in small pocket size electrochemical devices, which makes then attractive to be used in point-of-care applications.

\section{Experimental}

\subsection{Reagents}

The breast cancer protein CA 15-3 was obtained from MyBioSource. Guanidine hydrochloride, human serum from male AB clotted whole blood, potassium phosphate monobasic, potassium hexacyanoferrate(II) trihydrate, potassium hexacyanoferrate(III), proteinase $\mathrm{K}$ and oxalic acid anhydrous were supplied by Sigma-Aldrich. Sulfuric acid from Panreac, 2-aminophenol from ACROS, di-potassium hydrogen phosphate and acetic acid glacial from Merck were also used. Throughout the work ultra-pure water (resistivity $=18.2 \mathrm{M} \Omega \mathrm{cm}$ ) was used. The CA $15-3$ and hexacyanoferrate solutions were prepared in phosphate buffer (0.1 M, pH 7.4).

\subsection{Instrumentation}

Voltammetric measurements were carried out using an Autolab PGSTAT 204 potentiostat/galvanostat (Metrohm Autolab) and electrochemical impedance spectroscopic (EIS) analysis were performed using an Autolab PGSTAT 128N (Metrohm Autolab). The equipment was controlled by NOVA software (version 1.11, Metrohm Autolab). Screen-printed gold electrodes (Au-SPE, DropSens, DRP-C223BT) with gold working $(\mathrm{d}=1.6 \mathrm{~mm})$ and auxiliary electrodes and a silver reference electrode were used.

\subsection{MIP/NIP construction}

The optimized procedure for the MIP preparation was as follows: after the activation of the Au-SPE with $0.5 \mathrm{M} \mathrm{H}_{2} \mathrm{SO}_{4}$ (applying 5 cyclic voltammetric scans; potential range: $-0.2 \mathrm{~V}$ to $+1.3 \mathrm{~V}$, scan rate: $\left.100 \mathrm{mV} \mathrm{s}^{-1}\right), 2 \mu \mathrm{L}$ of a CA $15-3$ solution $\left(50 \mathrm{UmL}^{-1}\right)$ was placed on the working electrode of the Au-SPE and left to incubate $(20 \mathrm{~min})$. After gently rinsing the Au-SPE with a $0.1 \mathrm{M}$ phosphate buffer ( $\mathrm{pH} 7.4$ ) and subsequent drying, $40 \mu \mathrm{L}$ of a $10 \mathrm{mM} 2$-aminophenol solution was placed on the Au-SPE and the electropolymerization was performed (10 cyclic voltammetric scans; potential range: $-0.1 \mathrm{~V}$ to $+0.9 \mathrm{~V}$, scan rate: $100 \mathrm{mV} \mathrm{s}^{-1}$ ). The polymerization solution was then replaced with $40 \mu \mathrm{L}$ of $0.1 \mathrm{M}$ phosphate buffer ( $\mathrm{pH} 7.4$ ) and an additional 5 cyclic voltammetric scans, using the same parameters as before, were applied to remove unpolymerized monomer residues and to stabilize the polymer. Finally, the protein was removed from the imprinted polymer by placing $0.5 \mathrm{M}$ oxalic acid $(12 \mathrm{~h})$ and $0.1 \mathrm{M}$ phosphate buffer $(1 \mathrm{~h})$ on the Au-SPE. A control electrode (non-imprinted polymer, NIP) was also prepared. The procedure for the preparation of this NIP was the same as for the MIP but no CA 15-3 was used in this case.

\subsection{Electrochemical detection}

The interactions between CA 15-3 and the MIP and NIP were evaluated using a redox probe $\left(5 \mathrm{mM}\left[\mathrm{Fe}(\mathrm{CN})_{6}\right]^{3-/ 4-}(1: 1)\right)$. Electrochemical techniques such as cyclic voltammetry $(\mathrm{CV})$, differential pulse voltammetry (DPV) and electrochemical impedance spectroscopy (EIS) were used to characterize the MIP/NIP during the construction and optimization processes. The potential range in 
the $\mathrm{CV}$ was between $-0.2 \mathrm{~V}$ and $+0.6 \mathrm{~V}$ ( 6 scans at a scan rate of $\left.50 \mathrm{mV} \mathrm{s}^{-1}\right)$, and the potential range in DPV was between $-0.2 \mathrm{~V}$ and $+0.5 \mathrm{~V}$ (pulse amplitude: $50 \mathrm{mV}$, step potential: $2.5 \mathrm{mV}$ ). In EIS, a sinusoidal signal with a $10-\mathrm{mV}$ amplitude and a fixed potential of $0.2 \mathrm{~V}$ was applied in a frequency range between 0.1 and $10.000 \mathrm{~Hz}$ with 50 frequencies distributed logarithmically.

\subsection{Sample preparation}

First aliquots of human serum were spiked with different CA 153 concentrations and were then diluted ( $1: 1)$ with $0.1 \mathrm{M}$ phosphate buffer ( $\mathrm{pH}$ 7.4). The resulting solutions were analyzed directly using the above-mentioned electrochemical techniques.

\section{Results and discussion}

\subsection{Construction and operation of the sensor}

The overall process of the preparation of the MIP (Fig. 1) consisted of 3 different stages: (1) CA 15-3 immobilization through adsorption on the working electrode of the Au-SPE; (2) imprinting by forming a thin polymer film, through electropolymerization, around the adsorbed protein; (3) protein removal and creation of selective cavities in the polymeric film.

Preliminary voltammetric tests showed that CA 15-3 was not electrochemically active (i.e. no oxidation/reduction peaks were observed) at the Au-SPE. Therefore, the analyses were conducted using $\left.\left[\mathrm{Fe}(\mathrm{CN})_{6}\right)\right]^{3-/ 4-}$ as redox probe, measuring the differences between the signals before and after protein binding. The use of the sensor for CA 15-3 analysis consisted of 4 steps (Fig. 1): (4) measurement of the $\left.\left[\mathrm{Fe}(\mathrm{CN})_{6}\right)\right]^{3-/ 4-}$ signal when the polymeric cavities are unoccupied (after extraction); (5) incubation of the MIP/Au-SPE in CA 15-3 standard or sample solutions for a defined time; (6) measurement of the $\left.\left[\mathrm{Fe}(\mathrm{CN})_{6}\right)\right]^{3-/ 4-}$ signal after incubation, when the polymeric cavities are partiality occupied; (7) regeneration of the sensor by extracting CA 15-3 from the polymer.

\subsubsection{CA 15-3 adsorption}

To test the adsorption of CA 15-3 on conventional glassy carbon and gold electrodes, a solution of the protein was dropped on the surfaces of these electrodes. Based on the measurements of the signal of the redox probe before and after protein deposition it was observed that CA 15-3 did not adsorb on the glassy carbon surface but adsorbed on the gold surface.

To study the possible immobilization of CA 15-3 on the AuSPE, $3 \mu \mathrm{L}$ of CA $15-3$ solutions $\left(0-100 \mathrm{U} \mathrm{mL}^{-1}\right)$ were placed on the working electrode of the Au-SPE for $20 \mathrm{~min}$. Before and after the immobilization approach DPV and EIS analysis were performed (Fig. 2). In the DPV analysis (Fig. 2A and B) a marked decrease of the peak current intensity $\left(i_{p}\right)$ was observed for protein concentrations between $0-25 \mathrm{U} \mathrm{mL}^{-1}$. For higher concentrations (50 and $100 \mathrm{U} \mathrm{mL}^{-1}$ ) the $\mathrm{i}_{\mathrm{p}}$ remained constant. In the EIS analysis (Fig. $2 \mathrm{C}$ and $D$ ) an increase of the resistivity with the increase of protein concentration up to $50 \mathrm{U} \mathrm{mL}^{-1}$ was found. Therefore, it could be concluded that CA 15-3 was successfully adsorbed on the electrode. Based on these results a CA $15-3$ concentration of $50 \mathrm{U} \mathrm{mL}^{-1}$ was selected for the preparation of the MIP.

\subsubsection{Molecular imprinting of $C A 15-3$}

After the successful adsorption of the protein on the Au-SPE the molecular imprinting was performed by electropolymerization using 2-aminophenol as functional monomer. A $50-\mu \mathrm{L}$ aliquot of a $10 \mathrm{mM}$ 2-aminophenol solution (in phosphate buffer $0.1 \mathrm{M} \mathrm{pH} 7.4$ ) was placed on the Au-SPE and 10 consecutive cyclic voltammetric cycles between $-0.1 \mathrm{~V}$ to $+0.9 \mathrm{~V}$ were applied. A control NIP was also prepared using the same conditions, but without the protein
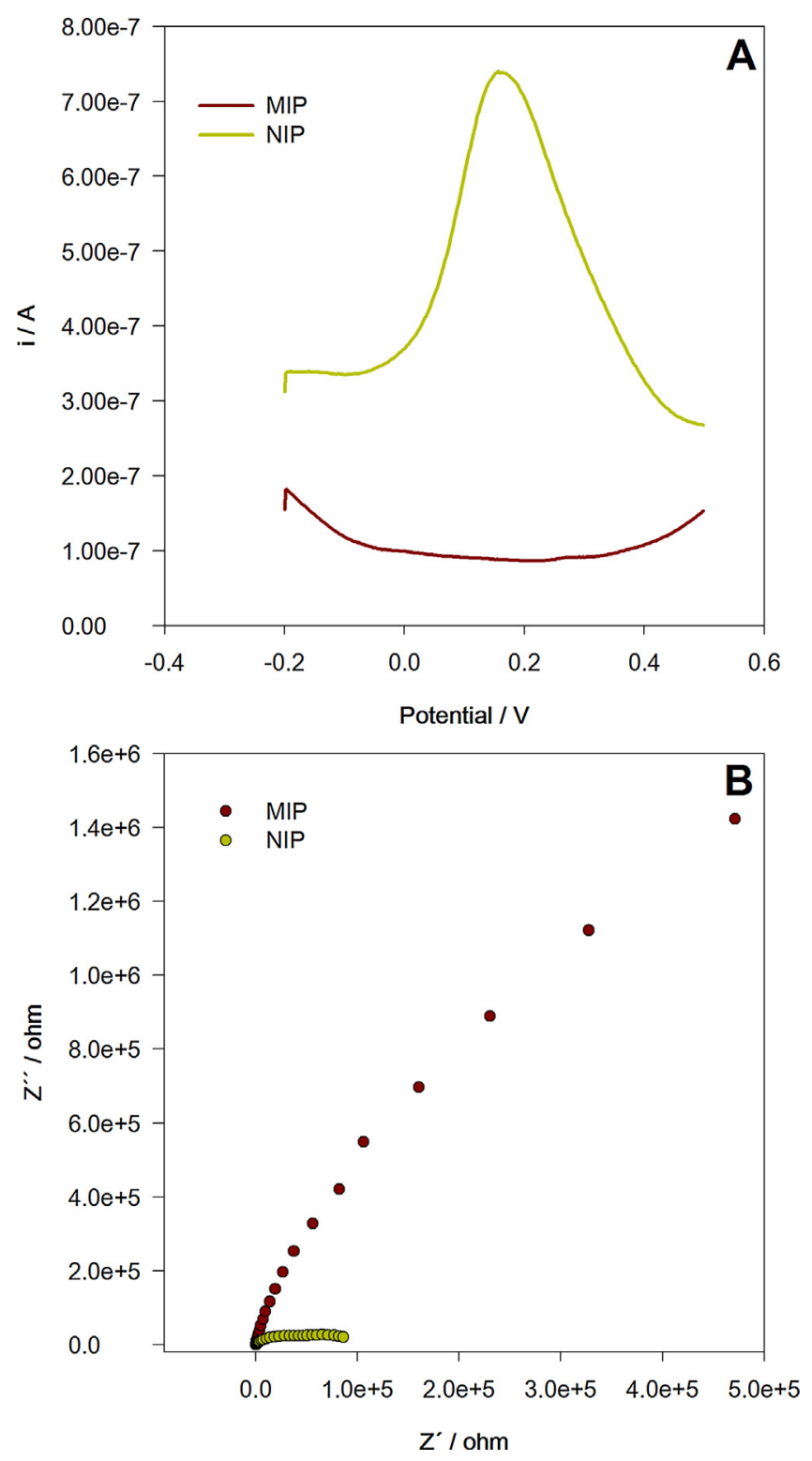

Fig. 4. Analysis of NIP/Au-SPE and MIP/Au-SPE after polymerization using $5 \mathrm{mM}$ $\left.\left[\mathrm{Fe}(\mathrm{CN})_{6}\right)\right]^{3-/ 4-}$. (A) DPV voltammograms and (B) EIS Nyquist diagrams.

adsorption step. The CVs corresponding to the preparation of the MIP and the NIP are shown in Fig. 3. There are clear differences between the electropolymerizations of the MIP and the NIP. In the NIP polymerization process the oxidation peak of 2-aminophenol was only observed during the first potential cycle. This indicated the formation of a nonconductive polymeric film at the surface of gold working electrode. In the MIP polymerization process the oxidation peak of 2-aminophenol was also observed during the first cycle, but a slower decrease of this peak was observed during the following cycles. This behaviour can be attributed to the presence of protein adsorbed on the surface of the gold working electrode, which slows the formation of the non-conductive polymer.

The prepared MIP and NIP were analyzed by DPV and EIS immediately after polymerization using a $\left.5 \mathrm{mM}\left[\mathrm{Fe}(\mathrm{CN})_{6}\right)\right]^{3-/ 4-}$ solution (Fig. 4). It can be confirmed that non-conductive polymer films were prepared with clear differences between the MIP and the NIP. The DPV analysis of the NIP showed a low $i_{p}$ of the redox probe while the EIS analysis revealed a high resistivity. However, for the MIP no oxidation peak was observed and a much higher resistivity was 

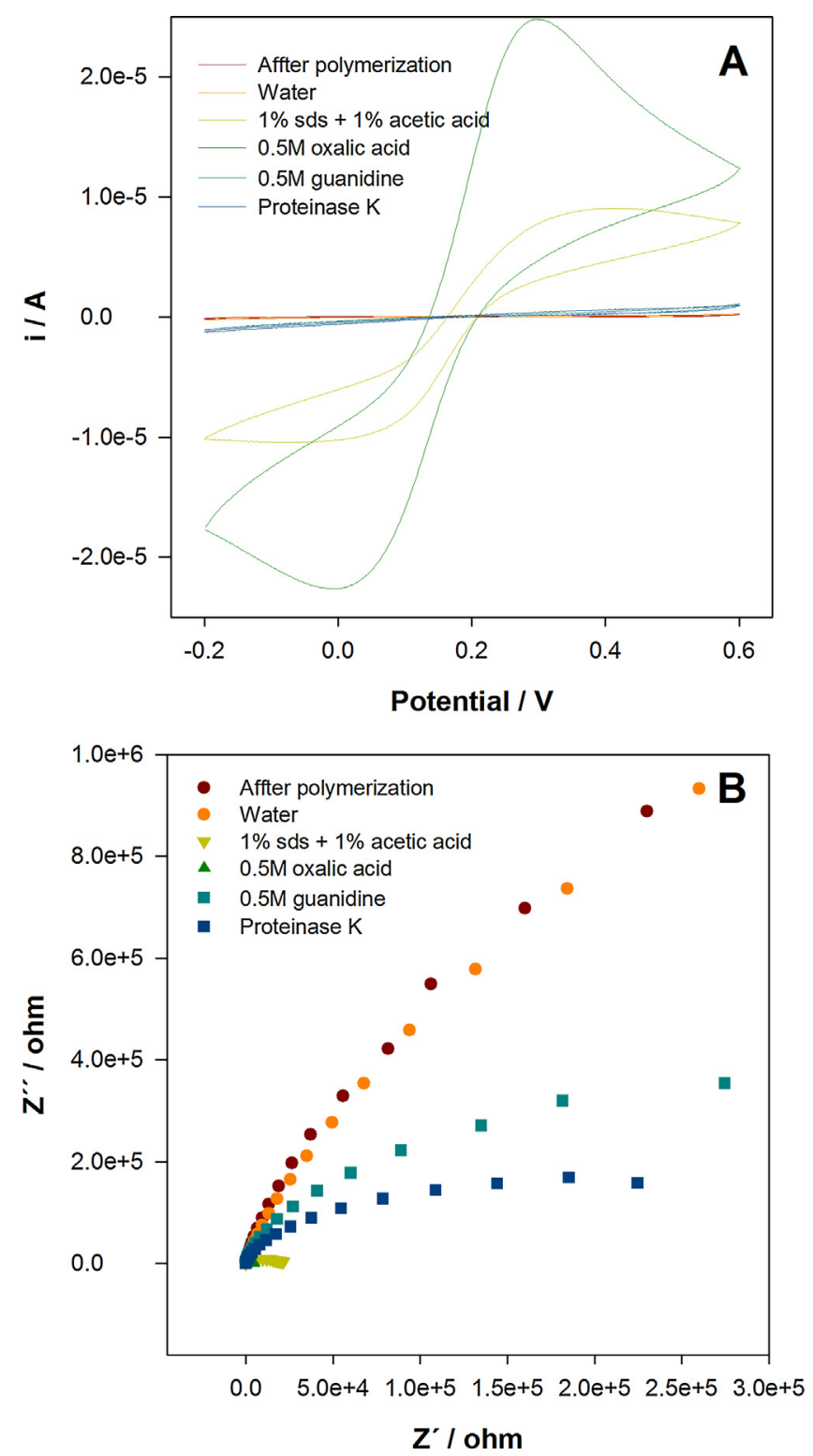

Fig. 5. Evaluation of the protein extraction using different solvents. (A) CV voltammograms and (B) EIS Nyquist diagrams obtained in $\left.10 \mathrm{mM}\left[\mathrm{Fe}(\mathrm{CN})_{6}\right)\right]^{3-/ 4-}$ and phosphate buffer $0.1 \mathrm{M} \mathrm{pH} 7$.

obtained. These results confirmed that the protein was successfully imprinted and entrapped in the polymeric film.

\subsubsection{Protein extraction}

An important step in the preparation of a MIP is the extraction of the template molecule after polymerization to create the specific binding cavities. The entrapped template molecules can be difficult to remove and is a problem that is frequently encountered in protein imprinting due to the size and complex structure of proteins. Therefore it is necessary to study the conditions that guarantee their efficient removal, which is most commonly accomplished by solvent extraction. For this purpose, and besides water, several aqueous extraction solutions were tested: $1 \%$ acetic acid + $1 \%$ SDS; $0.5 \mathrm{M}$ oxalic acid; $0.5 \mathrm{M}$ guanidine, and $0.5 \mathrm{M}$ proteinase K solutions. Five MIP sensors were prepared and after polymerization each of them was exposed to a different extraction solution overnight. Then the sensors were placed in phosphate buffer $(0.1 \mathrm{M}, \mathrm{pH} 7.4)$ for one hour and the extraction efficiency was evaluated comparing the CV and EIS analysis of the sensor before and after exposure to the sol-
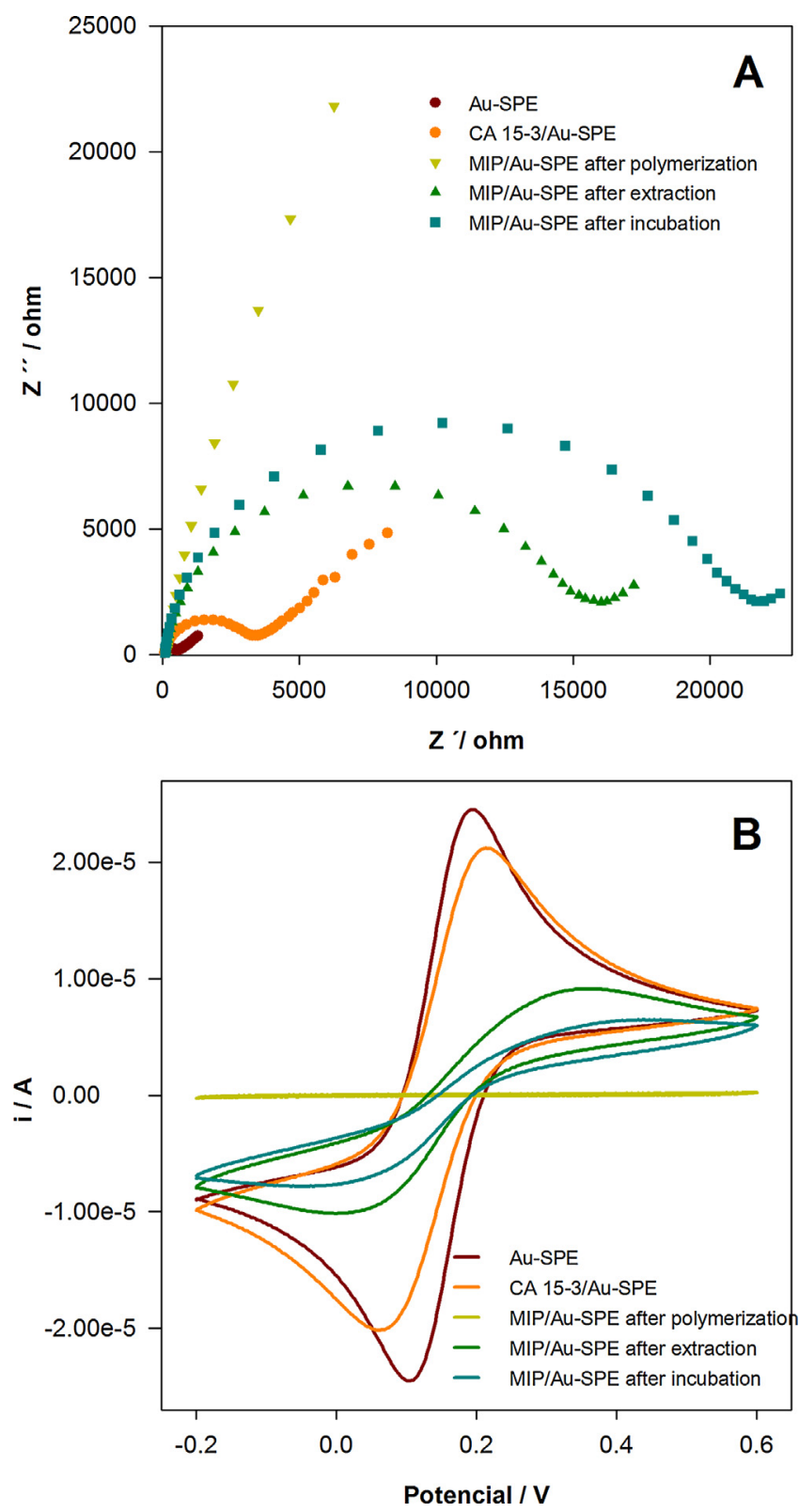

Fig. 6. Characterization of the step by step construction of the MIP/Au-SPE sensor (A) CV voltammograms and (B) EIS Nyquist diagrams in $\left.5 \mathrm{mM}\left[\mathrm{Fe}(\mathrm{CN})_{6}\right)\right]^{3-/ 4-}$ and $0.1 \mathrm{M}$ phosphate buffer $0.1 \mathrm{M} \mathrm{pH} 7$.

vents (Fig. 5). In the case of water no significant differences between the CV and EIS results before and after its use were observed. This indicates that the protein could not be extracted with water. In the case of the guanidine and proteinase K solutions, small variations in the CV analysis were seen, but no oxidation/reduction peak was obtained. In the EIS analysis a more pronounced difference was observed, but the resistivity remained high. The major differences were achieved using the acidic solutions. For both of them oxidation/reduction peaks in the $\mathrm{CV}$ analysis and a much lower resistivity in the EIS were observed after the extraction of the protein. These results can be attributed to the efficient extraction of the protein, and thus the formation of the specific cavities in the film. These cavities allow $\left.\left[\mathrm{Fe}(\mathrm{CN})_{6}\right)\right]^{3-/ 4-}$ to reach the surface of electrode which in turn results in oxidation/reduction peaks. The solution containing oxalic acid was chosen as extraction solvent since it produces the highest difference in the responses before and after extraction. 
A

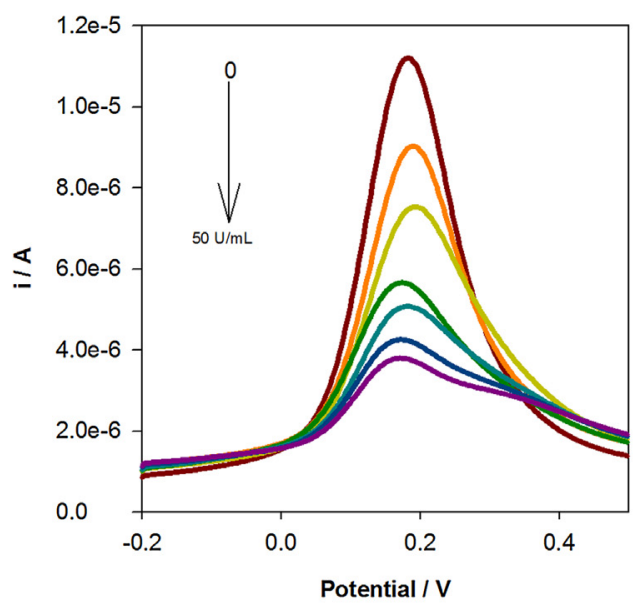

C

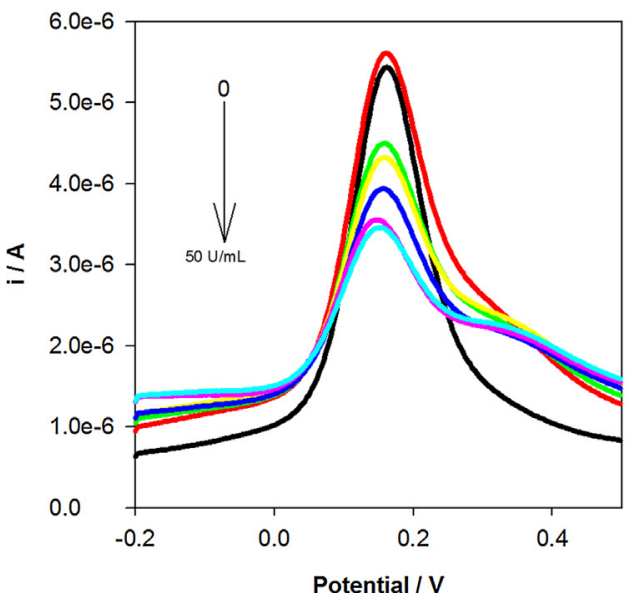

B

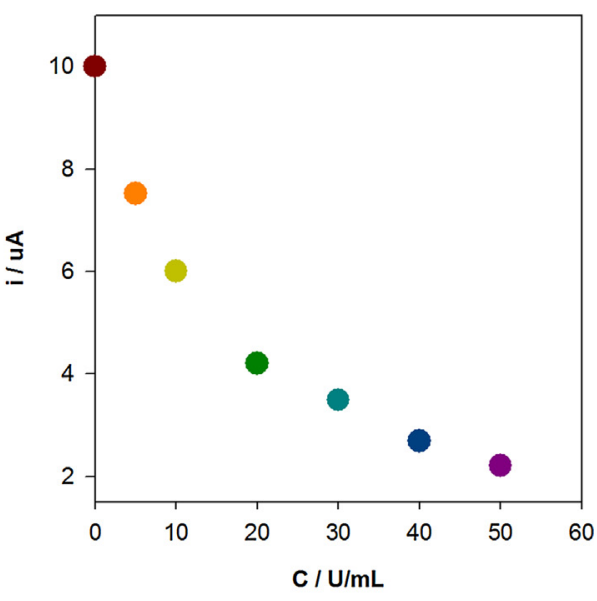

D

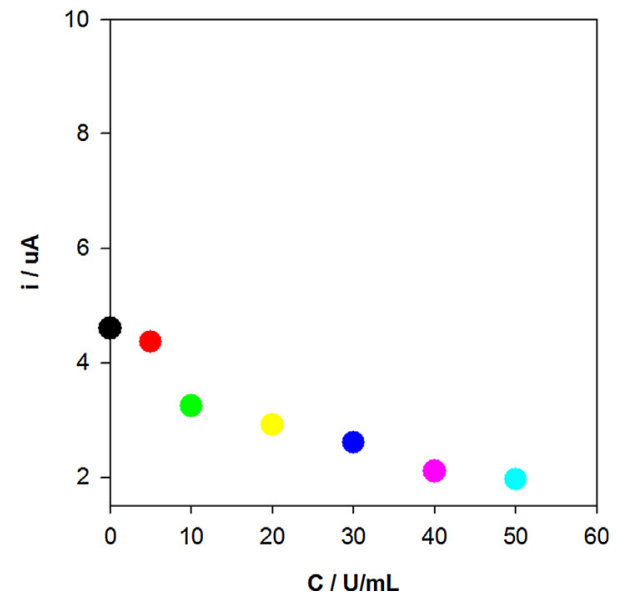

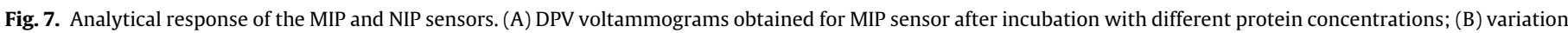

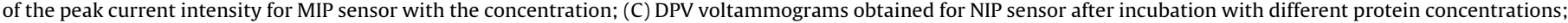
(D) variation of the peak current intensity for NIP sensor with the concentration.

\subsection{Sensor characterization}

During each step of the construction of the MIP and its subsequent use, the sensor was characterized by CV and EIS using $5 \mathrm{mM}$ $\left.\left[\mathrm{Fe}(\mathrm{CN})_{6}\right)\right]^{3-/ 4-}$ (Fig. 6). In EIS the semicircle of the Nyquist diagram for higher frequencies corresponds to the electron transfer process while the linear part of the lower frequency corresponds to diffusion. In Fig. 6(A) the straight line for the Au-SPE corresponds to a diffusion limited process with fast electron transfer kinetics. After the adsorption of CA 15-3 there is an increase of the semicircle, indicating a blocking effect caused by the presence of the protein that results in the reduction of electron transfer and an increase of the resistance to the electron flow. After polymerization there was a high increase of the impedance and the surface was highly resistant to electron transfer due to the formation of the polymer film containing the entrapped protein. After protein extraction a high decrease of the impedance was observed. This can be explained by the formation of cavities after protein removal, improving the diffusion of $\left[\mathrm{Fe}(\mathrm{CN})_{6}\right]^{3-/ 4-}$ through the polymer which facilitates the electron transfer electrons. Finally, after the incubation of the protein again a slight increase of the semicircle occurred, which indicates that the protein was successfully bound to the MIP and occupied some of the selective cavities.
Similar behaviors were observed in the CV analyses (Fig. 6(B)). Two well defined redox peaks were observed for the unmodified Au-SPE. After protein adsorption, a slight decrease of the peak current intensities occurred. After polymerization no redox peaks were obtained. This is a result of the blockage of the electron transfer by the polymer. After protein extraction the redox peaks once again appeared, indicating the efficiency of the extraction. As mentioned before, the extraction of the protein from the polymer creates cavities that increase the porosity of the MIP, which facilitates the diffusion of $\left[\mathrm{Fe}(\mathrm{CN})_{6}\right]^{3-/ 4-}$ to the electrode surface and the occurrence of the redox reaction. Finally, after incubation of the MIP sensor with the protein a decrease of the redox peaks was registered. This can be explained by the fact that the CA 15-3 molecules fill the cavities that block the diffusion of $\left[\mathrm{Fe}(\mathrm{CN})_{6}\right]^{3-/ 4-}$. It should be noted that there is a large difference in the EIS and CV analysis between unmodified Au-SPE and MIP/Au-SPE after extraction.

\subsection{Analytical response}

The analytical response of both the MIP and NIP sensors was tested. Solutions with different concentrations of CA 15-3 (5-50 $\left.\mathrm{U} \mathrm{mL}^{-1}\right)$ were incubated during $10 \mathrm{~min}$ and a DPV voltammogram was recorded (Fig. 7). 


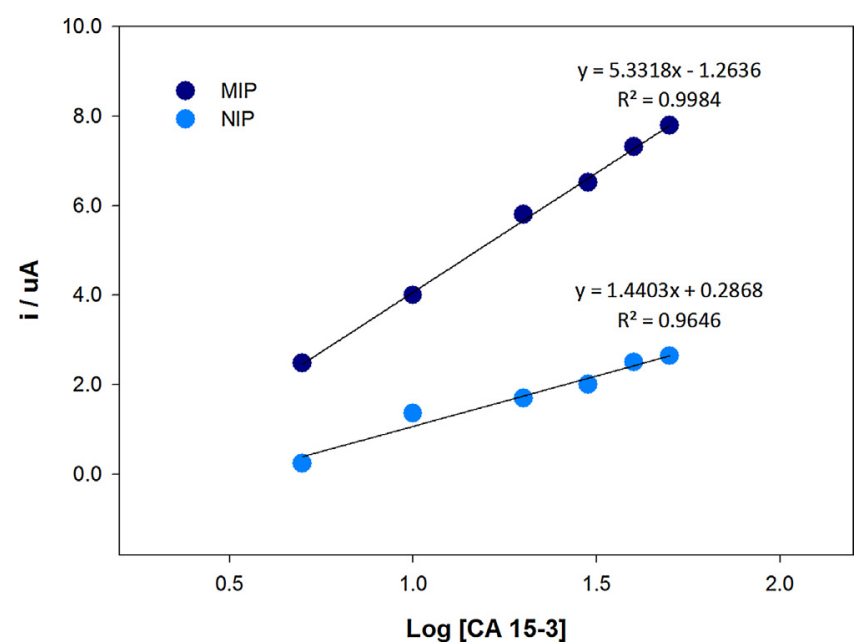

Fig. 8. Variation of $i_{p}$ with the logarithm of the CA 15-3 concentration for the MIP/Au-SPE and NIP/Au-SPE sensors.

A decrease of the $i_{p}$ of the redox probe was observed with the increase of the CA 15-3 concentration for both the MIP and NIP sensors. These results indicated that both sensors responded to variations of the CA 15-3 concentration. However, the decrease of the $i_{p}$ is more pronounced in MIP sensor. Therefore, the MIP sensor provides a much better sensitivity.

These results were used to construct calibration curves by plotting the current variation $\left(i_{p}\right.$ before incubation $-i_{p}$ after incubation) as a function of the logarithm of the CA 15-3 concentration (Fig. 8). The variation of the $i_{p}$ of the redox probe was linearly proportional to the logarithm of concentration of CA 15-3 in the tested range. The linear regression equation for the MIP sensor was $\mathrm{i}_{\mathrm{p}}(\mathrm{A})=5.33 \times \log [\mathrm{CA} 15-3]\left(\mathrm{U} \mathrm{mL}^{-1}\right)-1.26(\mathrm{r}=0.9984)$ and for the NIP sensor $i_{p}(A)=1.44 \times \log [C A 15-3]\left(U^{-1}\right)+0.28(r=0.9646)$.

The limit of detection (LOD) was calculated using the following equation: $L O D=3 \mathrm{~s} / \mathrm{m}$, where "s" is the standard deviation of the intercept and " $\mathrm{m}$ " is the slope of the calibration plot. The limit of detection (LOD) for the MIP sensor was estimated to be $1.5 \mathrm{U} \mathrm{mL}^{-1}$.

\subsection{Selectivity studies}

Another breast cancer biomarker, the extracellular domain of the human epidermal growth factor receptor 2 (HER2-ECD), and a biomarker of kidney function cystatin $C$ (CC) were selected to study the selectivity of the MIP sensor. Both are proteins that are smaller in size than CA 15-3. Solutions of these proteins were prepared in phosphate buffer with concentrations of $15 \mathrm{ng} \mathrm{mL}^{-1}$ and $570 \mathrm{ng} \mathrm{mL}^{-1}$ for HER2-ECD and CC, respectively. The responses of the MIP sensor to these proteins were studied and the results were compared with the MIP sensor's response to a solution of CA 15-3 $30 \mathrm{U} \mathrm{mL}^{-1}$ (Fig. 9). The peak current variation for HER2-ECD and CC corresponded to 55 and $24 \%$ of the CA $15-3$ peak variation, respectively. This indicates that HER2-ECD could have some interference in the analysis.

\subsection{Detection of CA 15-3 in human serum samples}

To validate its usefulness in complex samples, the MIP/Au-SPE sensor was applied to the detection of CA 15-3 in spiked human serum. For this purpose serum samples were spiked with three different protein concentrations: 10,30 and $50 \mathrm{U} \mathrm{mL}^{-1}$. Recoveries of 72,82 and $87 \%$ with relative standard deviations (RSD, \%, n=3) of 5,9 and $9 \%$ were obtained, respectively. These results proved the ability of the proposed sensor to be used as a fast, cheap, and easy to

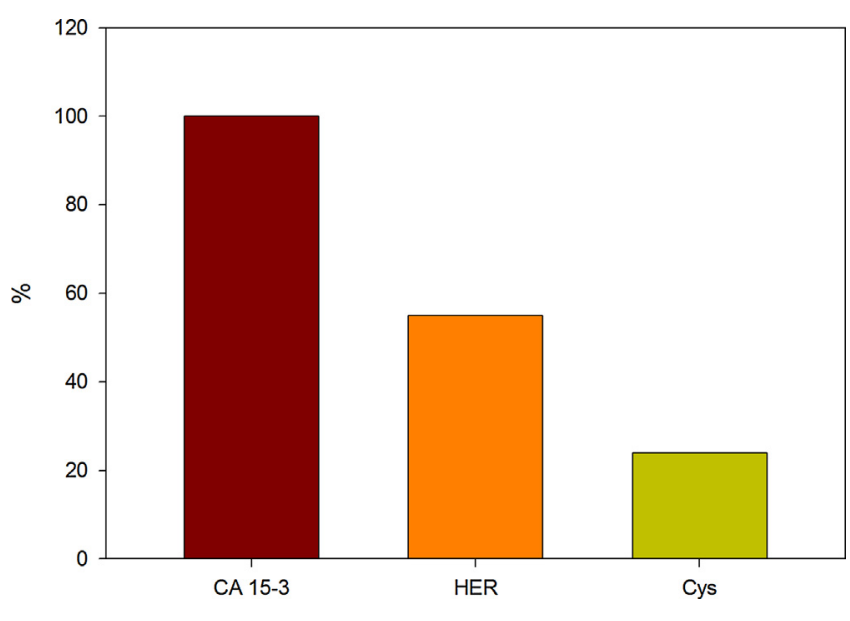

Fig. 9. Selectivity studies.

perform method for CA 15-3 analysis in biological samples without the need of laborious pre-treatment steps or methods.

\section{Conclusions}

In this work a gold screen-printed gold electrode was modified with a molecularly imprinted polymer for the detection of the breast cancer biomarker CA 15-3. A linear relationship between the peak current intensity of the hexacyanoferrate redox probe and the logarithm of the CA15-3 concentration was established between 5 and $50 \mathrm{U} \mathrm{mL}^{-1}$. The attained detection limit of $1.5 \mathrm{U} \mathrm{mL}^{-1}$ is well below the used cut-off value in clinical practice $\left(25 \mathrm{U} \mathrm{mL}^{-1}\right)$. Therefore, the developed sensor is promising for its use in the clinical setting. Besides this, the MIP sensor is an excellent candidate to be included in point-of-care devices because of the short analysis time (15 min) and, its simplicity and low cost, which could be very important in the monitoring of the patient's response to treatment and/or to evaluate the recurrence/progression of the cancer.

\section{Acknowledgements}

João Paulo Grosso Pacheco is grateful to FCT-Fundação para a Ciência e a Tecnologia for a Pos-Doc. grant (SFRH/BPD/101419/2014), financed by POPH-QREN-Tipologia 4.1-Formação Avançada, subsidized by Fundo Social Europeu and Ministério da Ciência, Tecnologia e Ensino Superior. Maria Freitas is grateful to FCT (Fundação para a Ciência e a Tecnologia) for her PhD grant (SFRH/BD/111942/2015). This work received financial support from the European Union (FEDER funds through COMPETE) and National Funds (FCT) through project UID/QUI/50006/2013. The authors are greatly indebted to all financing sources.

\section{References}

[1] International Agency for Research on Cancer, Cancer Fact Sheets: All Cancers Excluding Non-Melanoma Skin Cancer, 2013 http://gco.iarc.fr/today/home.

[2] World Health Organization, Cancer Fact Sheet N(297) [updated February] 2015 http://www.who.int/mediacentre/factsheets/fs297/en/\#.

[3] International Agency for Research on Cancer, Cancer Fact Sheets: Breast Cancer, 2013 http://gco.iarc.fr/today/home.

[4] E. Senkus, S. Kyriakides, S. Ohno, F. Penault-Llorca, P. Poortmans, E. Rutgers, et al., Primary breast cancer: ESMO clinical practice guidelines for diagnosis, treatment and follow-up, Ann. Oncol. 26 (2015) v08-v30.

[5] G.W. Sledge, E.P. Mamounas, G.N. Hortobagyi, H.J. Burstein, P.J. Goodwin, A.C. Wolff, Past, present, and future challenges in breast cancer treatment, J. Clin. Oncol. 32 (2014) 1979-1986.

[6] K.D. Cole, H.J. He, L. Wang, Breast cancer biomarker measurements and standards, Proteom. Clin. Appl. 7 (2013) 17-29.

[7] N.L. Henry, D.F. Hayes, Cancer biomarkers, Mol. Oncol. 6 (2012) 140-146. 
[8] J.F. Rusling, C.V. Kumar, J.S. Gutkind, V. Patel, Measurement of biomarker proteins for point-of-care early detection and monitoring of cancer, Analyst 135 (2010) 2496-2511.

[9] Z.J. Sahab, S.M. Semaan, Q.X. Sang, Methodology and applications of disease biomarker identification in human serum, Biomark. Insights 2 (2007) 21-43.

[10] J. Agbenyega, Molecular imprinted polymers, Mater. Today 12 (7) (2009).

[11] W.J. Cheong, S.H. Yang, F. Ali, Molecular imprinted polymers for separation science: a review of reviews, J. Sep. Sci. 36 (2013) 609-628.

[12] S.-W. Lee, S. Korposh, R. Selyanchyn, Fundamentals and perspectives of molecular imprinting in sensor applications, in: Handbook of Molecular Imprinting, Pan Stanford Publishing, 2012, pp. 3-63.

[13] M.J. Whitcombe, I. Chianella, L. Larcombe, S.A. Piletsky, J. Noble, R. Porter, et al., The rational development of molecularly imprinted polymer-based sensors for protein detection, Chem. Soc. Rev. 40 (2011) 1547-1571.

[14] Y.Q. Lv, T.W. Tan, F. Svec, Molecular imprinting of proteins in polymers attached to the surface of nanomaterials for selective recognition of biomacromolecules, Biotechnol. Adv. 31 (2013) 1172-1186.

[15] N.W. Turner, C.W. Jeans, K.R. Brain, C.J. Allender, V. Hlady, D.W. Britt, From 3D to 2D: a review of the molecular imprinting of proteins, Biotechnol. Prog. 22 (2006) 1474-1489.

[16] D. Cai, L. Ren, H.Z. Zhao, C.J. Xu, L. Zhang, Y. Yu, et al., A molecular-imprint nanosensor for ultrasensitive detection of proteins, Nat. Nanotechnol. 5 (2010) 597-601.

[17] X.W. Kan, Z.L. Xing, A.H. Zhu, Z. Zhao, G.L. Xu, C. Li, et al., Molecularly imprinted polymers based electrochemical sensor for bovine hemoglobin recognition, Sens. Actuators B: Chem. 168 (2012) 395-401.

[18] L. Li, L.L. Yang, Z.L. Xing, X.J. Lu, X.W. Kan, Surface molecularly imprinted polymers-based electrochemical sensor for bovine hemoglobin recognition, Analyst 138 (2013) 6962-6968.

[19] S. Viswanathan, C. Rani, S. Ribeiro, C. Delerue-Matos, Molecular imprinted nanoelectrodes for ultra sensitive detection of ovarian cancer marker. Biosens. Bioelectron. 33 (2012) 179-183.

\section{Biographies}

João G. Pacheco obtained his PhD in Chemistry in 2010 (Faculty of Sciences, University of Porto, Portugal). Currently he is a postdoctoral research in REQUIMTE and his present research interests include the development of analytical (electrochemical) methodologies based on the use of molecularly imprinted polymers for pharmaceutical, clinical, and food analysis.
Marta S.V. Silva obtained her BSc degree in Biomedical Engineering in 2012 and her MSc degree in Medical Computing and Instrumentation Engineering in 2015 (School of Engineering of the Polytechnic Institute of Porto (ISEP-IPP, Portugal)). She currently works in a medical and hospital equipment company (IBERDATA) as Systems Analyst.

Maria Freitas obtained her BSc degree in Biochemistry in 2010 (University of Trásos-Montes and Alto Douro, Portugal) and her MSc degree in Quality Control in 2012 (Faculty of Pharmacy, University of Porto, Portugal). Currently she is a PhD student in REQUIMTE and her present research interests include the development of analytical (electrochemical) methodologies for the analysis of cancer biomarkers.

Henri P.A. Nouws obtained his PhD in Chemistry in 2007 (Faculty of Sciences, University of Porto, Portugal). Currently he is an auxiliary professor at the Chemical Engineering Department of the School of Engineering, Polytechnic Institute of Porto (ISEP-IPP, Portugal). He is also an integrated research member of REQUIMTE and his present research interests include the development of analytical (electrochemical) methodologies for pharmaceutical, clinical, and food analysis.

Cristina Delerue-Matos obtained her PhD in Chemical-Physics, specialty in electrochemistry, in 1990. She is principal coordinator professor at the School of Engineering of the Polytechnic Institute of Porto (ISEP-IPP, Portugal) and also coordinates the REQUIMTE/ISEP research group (www.graq.isep.ipp.pt). Her research interests include the development of analytical methodologies for environmental, food, pharmaceutical, biochemical and industrial control. She is co-author of more than 250 publications in scientific journals. 